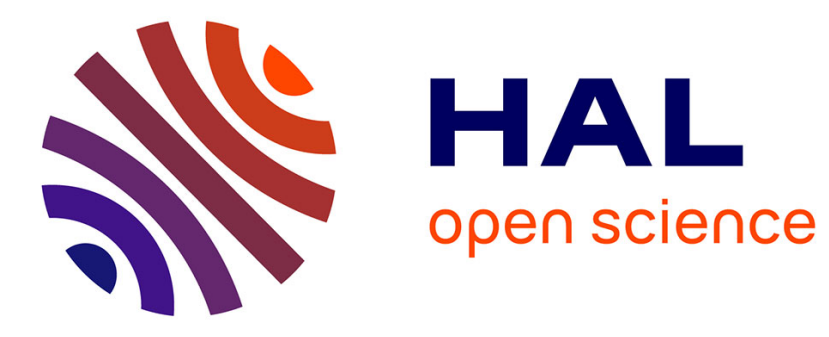

\title{
Migrants connectés, intégration sociale et apprentissage/certification en langues : prendre en compte la nouvelle donne numérique
}

Claude Springer

\section{- To cite this version:}

Claude Springer. Migrants connectés, intégration sociale et apprentissage/certification en langues: prendre en compte la nouvelle donne numérique. The linguistic Integration of Adult Migrants: some lessons from research - L'intégration linguistique des migrants adultes: les enseignements de la recherche, De Gruyter Mouton Open, 2017, 978-3-11-047747-4. 10.1515/9783110477498-006 . hal01540758

\section{HAL Id: hal-01540758 \\ https://hal.science/hal-01540758}

Submitted on 16 Jun 2017

HAL is a multi-disciplinary open access archive for the deposit and dissemination of scientific research documents, whether they are published or not. The documents may come from teaching and research institutions in France or abroad, or from public or private research centers.
L'archive ouverte pluridisciplinaire HAL, est destinée au dépôt et à la diffusion de documents scientifiques de niveau recherche, publiés ou non, émanant des établissements d'enseignement et de recherche français ou étrangers, des laboratoires publics ou privés. 
Claude Springer

\section{Migrants connectés, intégration sociale et apprentissage/certification en langues : prendre en compte la nouvelle donne numérique}

Résumé : Ce début du XXI ${ }^{\mathrm{e}}$ siècle est marqué par l'explosion des réseaux sociaux. La dimension sociale, que l'on peut déduire de la prise en compte de l'approche actionnelle mais également des réseaux sociaux, doit être interprétée dans sa globalité, à la fois physique et virtuelle. L'acteur social est aujourd'hui connecté, confronté à des « textes » qui ne relèvent plus uniquement du mode écrit et de la littératie classique, ni même de la plurilittératie. La question de l'intégration linguistique des migrants doit ainsi prendre en compte la nouvelle donne numérique pour pouvoir passer du migrant connecté à l'apprenant migrant connecté.

Abstract: The early 21st century has seen a proliferation of social networks. The social dimension, which is implicit in the focus on an action-based approach and also in the social networks, must be interpreted in its entirety, in both physical and virtual terms. Social agents nowadays go online and encounter "texts" which are no longer solely written and based on conventional literacy, nor on pluriliteracy for that matter. The linguistic integration of migrants must accordingly take into account the new digital environment so as to facilitate the transition from "online migrant" to online migrant learner.

\section{Entrée en matière : le migrant des poètes alsaciens}

L'Alsace, qui accueille plusieurs institutions européennes et ce symposium, offre un éclairage particulier à la question qui nous préoccupe, celle de l'intégration linguistique des migrants adultes. De manière très symbolique, Les Serments de Strasbourg de 842 instituent un certain bilinguisme avec le français et l'allemand, ou plus exactement le proto français, le gallo-roman, et le proto alle-

Claude Springer, professeur émérite, Université Aix-Marseille, claude.springer@univ-amu.fr

DOI 10.1515/9783110477498-006 
mand, le francique rhénan. Les Serments sont pour moi emblématiques d'une Alsace multilingue avec l'alsacien comme langue identitaire et langue d'un tiersespace entre la France et l'Allemagne. L'histoire tragique des guerres a marqué cette région qui, comme d'autres, s'est vue assimilée malgré elle aux pays qui la convoitaient.

Les artistes et poètes alsaciens ont exprimé à leur façon une autre approche de la langue et de l'identité.

\subsection{Jean Hans Arp (1886-1966) : l'indicible et l'indétermination}

Il a connu les deux guerres mondiales. Il est né, comme il aime le dire, de la nature, il est né de Strasbourg, il est né d'un nuage : " Ich bin in Strassburg geboren. Ich bin in einer Wolke geboren ». La détermination indéterminée de son origine alsacienne montre la difficulté à se définir par une nationalité, par une ville particulière. Son lieu de naissance, l'Alsace ne serait pour lui qu'une potentialité a-territoriale, un espace flottant difficile à situer. La sculpture et la poésie sont intimement liées à sa vie et à sa philosophie. Elles sont complémentaires comme les trois langues qu'il parle. Une des sculptures de Jean Hans Arp, Nombril et deux idées, représente, pour moi, un corps disloqué par la guerre. La guerre est un Wahnsinn, une folie, qu'il exprime à travers l'Unsinn, le nonsens que la poésie permet d'exprimer. Le nombril est une figure poétique qui représente l'origine, le centre de la vie, de l'intime mais aussi le centre du langage, l'origine du nom. Ce nom est vide et disparait dans le néant. Le nombril symbolise aussi les entrailles du poète, l'univers du rêve, de sa langue maternelle, l'alsacien, langue de l'enfance. Les deux idées qui sont posées maladroitement sur le corps peuvent représenter ses deux langues apprises scolairement. Il y a du Jean et du Hans à la fois.

Les poèmes de Jean Hans Arp ne sont pas très connus (Arp, 2014). Die Grosse Firgelei, s'intéresse à la langue, façon dada, dont il fut un des promoteurs. Il montre la part d'indicible et d'incompréhensible de toute langue. La part du rêve, du nuage, de l'intime. La langue ne saurait être réduite à son seul usage communicatif fonctionnel. Je me permets cette glose : Firgelei m'a dit textuellement ce qui suit, dit le poète. Une langue est belle si elle permet de s'attarder et de rêver, de penser et de fantasmer. Une langue est belle si elle chante des fleurs de rêve. Seule la langue de l'enfance rend possible ces rêveries, la langue fonctionnelle en est incapable. Dans le poème Seither spaziere ich, Depuis lors je me promène, Jean Hans Arp pose la question de l'identité : qui suis je ? d'où suisje ? Il y a cette impossibilité de faire son unité. Cet autre que l'on cherche est 
parti loin, dans le pays des rêves. Il est devenu inaccessible. Il n’y a plus moyen de lui poser les questions qui taraudent. On ne peut plus savoir qui on est et d'où on vient.

Ces poèmes de fin de vie montrent l'autre aspect de la langue que les linguistes ignorent malheureusement. La langue n'est pas qu'un outil pour communiquer, pour se débrouiller dans les situations quotidiennes. Elle est aussi poésie, rêverie, imaginaire. Le poète nous parle des conséquences de l'assimilation et de notre modernité, qui ne nous permet plus réellement de nous définir par une origine nationale et culturelle. Le nouvel homme que nous devenons finit par être un étranger, un migrant. Il vit dans un nouvel espace, un tiers espace, et a du mal à renouer les fils de son origine. Les souvenirs lointains font désormais partie du Traumheitland, du pays des rêves.

\subsection{André Weckmann (1924-2012) : violence de l'assimilation}

André Weckmann, est né après la $1^{\mathrm{e}}$ guerre mondiale à Steinbourg. Il a écrit plusieurs recueils de poèmes en alsacien (Huck, 1989). Le premier poème Sproch définit la langue. La langue, pour le poète, est faite des choses simples de la vie. La langue c'est la vie, dans sa matérialité et sa banalité. C'est surtout les histoires d'une vie, histoires qui fondent notre généalogie, qui remonte à notre origine. La langue s'inscrit ainsi dans l'histoire d'un lieu, dans les histoires que partage une communauté. Langue et identité sont intimement liées et se nourrissent des liens que nous tissons avec les autres. Le regard du poète sur la banalité de la vie nous fait comprendre la dimension sociale de la langue. Le deuxième poème sur les Alsaciens, Älsässer, rappelle les douleurs d'une région déchirée par les guerres, le non-sens de la nationalité qui fait que l'on peut être selon les moments de l'histoire l'ennemi des uns et des autres et se faire tuer par les uns ou les autres. L'Alsacien est comme un apatride dans son propre pays, un étranger qui ne sait pour qui et pour quoi il va encore se faire pendre.

Ces deux poèmes questionnent de la même façon l'idée de nation, de nationalité, d'identité et d'intégration linguistique. L'intégration linguistique ressemble à une dépossession culturelle si on considère que la langue est bien celle de l'origine, celle des rêves et de la création, celle de l'intime fantasmé. 


\subsection{Premières impressions : Langue et intégration}

La vision de la langue qui est exprimée par les poètes est loin de celle qui ressort de nos analyses linguistiques et didactiques. Nous avons la fâcheuse manie à ne prendre en compte que les simples mécanismes linguistiques et communicatifs. La langue est avant tout la vie, la vie simple de tous les jours, une façon de «se donner la main ». Mais c'est aussi, la poésie, l'imaginaire de nos histoires, des " fleurs de rêve », que l'on offre. Tout peut basculer, soudainement, nos repères et nos mots peuvent être désintégrés : " e hass " peut d'un coup devenir " un lapin », pour reprendre un autre poète alsacien Claude Vigée (2000), par le simple jeu de l'intégration/assimilation linguistique. Les migrants vivent cette violence et questionnent la langue, l'identité, l'assimilation. Les regards des poètes alsaciens nous permettent de mieux comprendre la violence de l'assimilation/intégration mais aussi les liens indestructibles qui lient langue et identité. Et, en fin de compte, ne faut-il pas admettre que l'image du migrant est celle de l'humanité ?

Amin Malouf résume bien cette première approche (1998) :

«Sans doute mes propos sont-ils ceux d'un migrant, et d'un minoritaire. Mais il me semble qu'ils reflètent une sensibilité de plus en plus partagée par nos contemporains. N'est-ce pas le propre de notre époque que d'avoir fait de tous les hommes, en quelque sorte, des migrants et des minoritaires?

Nous sommes tous contraints de vivre dans un univers qui ne ressemble guère à notre terroir d'origine ; nous devons tous apprendre d'autres langues, d'autres langages, d'autres codes ; et nous avons tous l'impression que notre identité, telle que nous l'imaginions depuis l'enfance, est menacée.

Beaucoup ont quitté leur terre natale, et beaucoup d'autres, sans l'avoir quittée, ne la reconnaissent plus. »

Les poètes, les artistes, les migrants ressentent avec force ces aspects universels de la condition humaine, de la modernité, de la migration.

\section{Faire évoluer notre vision du migrant adulte : le migrant connecté}

La migration au XXI ${ }^{\mathrm{e}}$ siècle ne peut se penser en dehors d'une société globalisée et interconnectée. Elle s'inscrit bien entendu dans cette histoire éternelle des guerres et des conquêtes qui débouchent inexorablement sur des déplacements de populations. Beaucoup d'études sur les migrants s'inscrivent logiquement dans cette vision classique et universelle de la migration. La plupart ignorent 
cependant l'évolution introduite par la société mondialisée du savoir numérique interconnecté et des réseaux sociaux. Il nous faut reconsidérer l'image que nous avons du migrant adulte déraciné au profit d'une image conforme à l'homme d'aujourd'hui qui est interconnecté et interagit sur les réseaux sociaux. Je propose d'explorer trois pistes. La première renvoie à la complexité qui inclut l’imaginaire et à la poésie ; la deuxième au paradoxe de la proximité et de la distance et la troisième à la société interconnectée.

\subsection{Imaginaire et poésie de la langue et de la vie}

Morin (2015: 30) propose de prendre en compte les deux polarités de l'homme. Pour lui, l'homme ne se réduit pas au prosaïque, au rationnel, au fonctionnel. On ne peut réduire les besoins vitaux aux seuls besoins de communication pour le quotidien. L'homme a également besoin de s'évader, besoin d'imaginaire, besoin de poésie.

La polarité prosaïque de la vie commande tout ce que nous faisons par contrainte, pour survivre, pour gagner notre vie. Et il y a la polarité poétique de la vie, c'est-à-dire celle où l'on s'épanouit personnellement, où l'on vit en communion, où l'on a des moments d'harmonie et de joie. Moments que donnent l'amour, l'amitié, la liesse. C'est cela qui est vivre, vivre poétiquement.

Cette part d'indicible fait partie du tout social, des interactions entre les individus, des histoires personnelles, des mondes symboliques qui constituent ce que l'on nomme la culture. L'homme peut se concevoir, certes, comme un être rationnel, mais il est aussi une personne émotionnelle qui s'inscrit dans une histoire de vie en mouvement. L'idée qu'il existerait une langue de survie, garantissant l'intégration sociale dans une société d'accueil, réduit le migrant à la seule polarité prosaïque de la vie. Cette optique a pour conséquence de focaliser la formation sur les situations quotidiennes de survie oubliant l'indispensable besoin d'imaginaire et de communion. Le descriptif du français langue d'intégration en France, par exemple, s'inscrit explicitement dans cette vision réductrice et présente les urgences du quotidien auxquelles on ajoute « des petits riens relationnels ». Or, justement, ces petits riens relationnels constituent le tout des langages sollicités et expriment le vivre poétiquement. La langue est aussi et surtout le lieu symbolique du social, le lieu de l'imaginaire poétique. 


\subsection{Le paradoxe de la distance et de la proximité}

La figure de l'étranger de Simmel (1908) permet d'envisager le migrant différemment. L'étranger fonctionne comme un symbole des relations humaines, mais aussi comme un symbole de la distance et de la mobilité, dans son aspect relationnel ou imaginaire. Il y a ainsi un paradoxe dans la mesure où l'étranger est à la fois ici et ailleurs, mais aussi dans un tiers espace, un iciailleurs, qu'il construit en permanence. La mobilité de l'étranger s'oppose à la sédentarité, l'étrangeté à la familiarité. Et en fin de compte, l'étranger est en nous. Nous pouvons nous sentir étrangers chez nous, lorsque nous allons vers d'autres communautés, lorsque nous avançons en âge en observant nos enfants, etc., comme nous avons déjà pu le dire.

Raphaël (2008:11) résume cette figure de l'étranger de la manière suivante : « cette ambivalence dans les rapports à l'étranger ne rejoint-elle pas ce qui caractérise toute relation qu'une personne entretient avec son entourage, et qui porte toujours la marque de la distance et de la proximité ? Tout homme fait l'expérience contradictoire de l'unité de son être et, en même temps, d'une faille, d'une certaine étrangeté à lui-même. » Pour Raphaël, il s'agit bien de « la forme exacerbée de toute relation humaine ». Il reprend la formule de Simmel : « La distance à l'intérieur de la relation signifie que le proche est lointain, mais le fait même de l'altérité signifie que le lointain est proche ».

Le migrant possède ainsi un capital social riche d'expériences dans diverses communautés. On ne peut que constater que la plupart des réflexions et des propositions de formation pour les migrants font l'impasse sur cette réalité complexe en se concentrant uniquement sur l'ici du pays d'accueil et sur un système artificiel d'interactions fonctionnelles, prosaïques pour reprendre Morin.

\subsection{Des réseaux de liens sociaux interconnectés}

De nombreuses études s'intéressent aux réseaux de liens que tissent les migrants dans leur aventure. Le monde d'internet ne connait pas les frontières et les barbelés que l'on érige pour freiner les migrants dans leur quête d'un meilleur lieu de vie. Diminescu (2005 : 277) reprend la réflexion de Simmel et estime que « mobilité et connectivité forment désormais un ensemble de base dans la définition du migrant du XXI siècle ». Elle résume cette nouvelle approche de la manière suivante : «Hier : immigrer et couper les racines ; aujourd'hui : circuler et garder le contact. Cette évolution semble marquer un nouvel âge dans l'histoire des migrations : l'âge du migrant connecté. » Il s'agit donc de « Considérer 
le migrant dans la totalité de ses mobilités (physiques, imaginaires, virtuelles) » (Diminescu 2005 : 278). L’intégration sociale n'est pas le fait de l'institution, c'est une forme d'intégration par le bas, nous dit Diminescu.

Bashi (2007) développe la même analyse au sujet des migrants de la Caraïbe, en référence aux liens forts et faibles de Granovetter (1973). Elle montre l'intérêt de l'étude des réseaux de liens qui caractérisent l'homme moderne et tout migrant. La sociologie transnationale montre que la migration n'est pas un simple déplacement spatial d'un lieu d'origine vers un lieu d'arrivée avec une obligation d'acculturation vers le lieu d'arrivée. Le processus migratoire doit être pris dans sa complexité et sa richesse. Le migrant est un acteur social qui vient avec un projet de vie. Il est en relation constante avec des personnes ressources, dans une toile aux liens multiples. Ces nœuds d'entraide constituent le tiers espace de la migration et permettent au migrant de trouver une place. La survie du migrant dépend de ces carrefours de liens sociaux qui se nouent et se dénouent entre lieu d'origine et lieu d'accueil. Notre vision sociologique du migrant comme personne déracinée, inculte, sans projet, doit impérativement être modifiée pour prendre en compte les points d'appui que les migrants utilisent.

\subsection{Une société interconnectée}

Siemens (2004) définit le nouveau courant du connectivisme et présente les principes de l'apprentissage avec le numérique de la manière suivante : l'apprentissage est chaotique, il est continu, il est complexe, il consiste à réaliser des connexions entre des informations en flux continu. L'apprentissage est une cocréation qui se réalise dans des communautés de pratiques. Il émerge des multiples interactions, dans les liens que la toile tisse en permanence. L'intelligence ne peut plus être uniquement représentée dans un cerveau individuel. Elle se développe aussi dans un réseautage collaboratif. De nouvelles formes d'apprentissage et de socialisation se développent selon une approche collaborative, de réciprocité, comme c'est le cas du mouvement Do it yourself ou Do it together.

Par ailleurs, Granovetter (1973) montre que chaque individu dispose de différents réseaux de liens sociaux. Le réseau intime, celui des amis sur les réseaux sociaux, est caractérisé par le temps passé ensemble à échanger, par l'intime fondé sur la confiance mutuelle et la réciprocité, comme l'avait défini Simmel. Ce réseau de l'intime constitue la forme première de sociabilité : ce sont les liens forts. Cependant, un deuxième type de réseau doit être pris en considération. Il est constitué de liens dits faibles. Ces liens forment un pont vers d'autres communautés, d'autres réseaux. Ils sont indispensables dans la mesure 
où ils permettent de faire circuler de nouvelles informations ; c'est le cas par exemple du réseau professionnel, du réseau autour de centres d'intérêts. Granovetter montre qu'un individu a plus de chances de trouver du travail s'il dispose de liens hors de son réseau intime, l'information étant alors plus diversifiée.

\subsection{Premier bilan : quelles conséquences?}

Il est urgent de questionner la vision institutionnelle de la politique migratoire et d'intégration, mais aussi notre vision sociologique du migrant comme personne déracinée et inculte. Le migrant adulte aujourd'hui doit être reconnu dans cette nouvelle dimension communautaire qui caractérise tout individu à l'ère du numérique.

Nous pouvons avancer la définition suivante. Le migrant est un individu mobile qui se déplace d'un endroit à un autre, porteur d'un projet. Il est donc ici et ailleurs à la fois, dans un espace hybride, un iciailleurs. Les artéfacts numériques accompagnent cette mobilité qui n'est plus uniquement spatiale et géographique mais aussi virtuelle et imaginaire. Il n'y a plus une démarcation nette entre l'ici et l'ailleurs, comme on le pensait en opposant réel et virtuel, ou société d'accueil et société d'origine. Le migrant connecté continue à développer des liens forts avec sa communauté d'origine et noue aussi d'autres liens vers de nouvelles communautés. En tant qu'acteur social, il produit des objets culturels qui illustrent une co-construction symbolique hybride caractérisant la richesse de la migration. La formation des migrants repose aujourd'hui malheureusement sur une approche sociologique qu'il nous faut dépasser.

\section{Présentation du contexte : Marseille}

Le contexte de Marseille va nous permettre d'illustrer cette nouvelle sociologie transnationale. La ville est en effet représentative de l'histoire des migrations et des difficultés d'intégration. La communauté comorienne de Marseille s'inscrit bien dans l'évolution que nous venons de décrire. Les associations marseillaises réalisent un travail indispensable pour permettre et faciliter la solidarité. Malheureusement, les nouvelles orientations législatives pour les migrants, qui intègrent les propositions européennes pour l'intégration linguistique, ont pour conséquence de renforcer une vision caricaturale et dépassée de la migration. Elles ignorent ces gisements indispensables de cohésion sociale. 


\subsection{Sociologie de Marseille}

Peraldi, Duport et Samson (2015) décrivent la complexité sociologique de Marseille. Marseille est à la fois le port de la Méditerranée synonyme de voyage, de commerce, de transit et la porte de la Méditerranée synonyme d'accueil, de communautés, de métissages culturels. Marseille a connu de nombreux flux migratoires, venus de l'Europe, en particulier les Italiens qui ont constitué une communauté importante dans le quartier du Panier (celui de la série télévisée Plus belle la vie). On se souvient aussi des Arméniens qui ont fui le génocide dans les années 1910/20. Ils furent plus de 70000 à venir à Marseille par la mer. Les Maghrébins constituent une communauté très importante. Ils sont venus à différentes périodes et en particulier après la décolonisation. Faut-il rappeler les 500000 rapatriés d'Algérie ? Les Africains sont également très présents, comme d'autres communautés.

Marseille a une image ambivalente avec d'un côté celle du melting pot, forte du multiculturalisme et du vivre ensemble, et de, l'autre côté, celle de violence, de délinquance proche du Chicago des années 1960. La ville est constituée de quartiers plus ou moins mélangés, avec une mobilité entre les quartiers nord, qui accueillent les migrants, et les quartiers sud où se trouvent les plages. Marseille Espérance fut créée en 1990 pour réunir toutes les communautés religieuses : communauté arménienne, communauté bouddhiste, communauté catholique, communauté juive, etc. Cet exemple d'approche communautariste permet de comprendre la spécificité marseillaise qui se distingue de l'approche républicaine et laïque d'autres villes françaises.

Marseille est riche aussi des cultures qui se créent au fil du temps dans ce laboratoire social qu'est la ville, dans ce tiers espace qui rend possible la création artistique. La poésie du rap et la musique ethnique permettent aux migrants sédentarisés de faire entendre leurs voix. L'art est une forme importante de la socialisation des jeunes et le rap marseillais est très vivant. Il est largement installé dans les quartiers nord et parle du mal être de la jeunesse et des désillusions de vivre dans une société qui promet l'égalité mais les stigmatise à cause de la couleur de leur peau et de leur religion. On comprend que la maitrise linguistique n'est pas porteuse d'intégration, que les diplômes de l'école ne procurent pas du travail. Il y a donc bien un échec du système d'intégration linguistique des migrants comme l'exprime Soprano, chanteur comorien de Marseille. Le migrant, basané et musulman, pourtant né en France, reste un immigré, un outsider, un looser, un exclu, un paria, un voyou. Les objets culturels, dont la mode ethnique, la cuisine, le sport, sont les emblèmes de la créativité de ces communautés. Ils constituent la diversité et la richesse du patrimoine culturel français. 


\subsection{Un exemple parmi d'autres : la communauté comorienne de Marseille}

L'exemple de la communauté comorienne de Marseille (il y a plus de Comoriens à Marseille qu'à Moroni) illustre l'opposition entre une sociologie de l'immigration, qui développe la vision de l'immigré déraciné, propre au courant républicain universaliste dominant en France, et une sociologie de la mobilité et du transnationalisme. L'approche de la mobilité s'intéresse aux nouvelles formes collectives d'appartenance sociale, dont les intérêts, les valeurs et les comportements dépassent les frontières. Le migrant, en tant qu'acteur, est porteur d'un projet migratoire. Il est citoyen du monde et s'intéresse à la politique de son pays qui lui permet de voter et de participer au destin de son pays, alors même que le pays d'accueil lui refuse cette participation citoyenne. La communauté comorienne est très active sur internet. Des associations se retrouvent sur Facebook et dévoilent la vie sociale au quotidien dans toute sa banalité. On y trouve tous les ingrédients de l'iciailleurs. Et pourtant, les principales propositions de formation linguistique ignorent cette richesse. Elles ne prennent en compte que la seule vision de l'insertion vers le pays d'accueil. Elles ignorent la réalité sociale du migrant connecté. Pourquoi taire la créativité que l'on trouve dans les nombreux objets culturels de la migration ? Pourquoi refuser de voir cette intelligence collective, ces lieux d'apprentissage formels et informels ?

\subsection{Quelques exemples du tissu associatif : l'association Mot à Mot et Femmes d'ici et d'ailleurs}

La Friche de la Belle de Mai à Marseille est un concentré d'associations. L'association Mot à Mot s'est installée dans des locaux associatifs partagés. Elle vise la formation linguistique des migrants ( $90 \%$ de femmes). Elle est dirigée par trois femmes permanentes, salariées (titulaires d'un master français langue étrangère). Les salaires proviennent de divers financements qui ne relèvent pas des fonds pour les migrants.

L'association s'inscrit dans les valeurs de l'éducation populaire qui reconnait à chacun la volonté et la capacité de progresser et de se développer. C'est une vision citoyenne et humaniste de la formation qui débouche sur une gestion participative de l'association.

Le circuit officiel pour les migrants ne couvre pas toute la demande de formation. Mot à mot s'adresse ainsi aux migrants qui échappent au circuit officiel, que ce soit pour l'alphabétisation ou le français langue étrangère. L'association propose aussi des ateliers passerelles pour aider les migrants à 
passer le permis de conduire (comprendre le code de la route), à accompagner les mères pour comprendre l'école et mieux suivre les enfants, pour développer les capacités artistiques, etc. Il n'est pas difficile de comprendre que ces associations, même réduites dans leurs ambitions et possibilités financières, jouent un rôle indispensable dans l'accueil et l'accompagnement des migrants. Elles sont mises en danger par la rationalisation de la commande et de l'offre institutionnelles pour les migrants.

L'association Femmes d'ici et d'ailleurs, créée en 1994, a pour objectif d'accompagner les femmes qui vivent en marge de la société. Il s'agit d'entraide entre femmes intégrées et femmes qui viennent d'arriver. Les locaux sont vastes et adaptés aux objectifs de l'association. L'association vise l'autofinancement. Elle fait partie d'un réseau d'associations locales et internationales qui sont tournées vers l'économie solidaire. La formation linguistique fait partie de la demande des personnes, en particulier l'alphabétisation. Cependant, ce n'est qu'un élément d'une approche fondée sur la définition d'un projet personnel avec une mise en valeur des compétences et de l'expertise de chaque personne. Pour cette association, il est indispensable de partir des savoir-faire de la personne et de voir comment ils peuvent être développés pour s'adapter à la réalité du marché. Il s'agit de faire émerger des projets personnels et de montrer qu'ils sont réalisables. La cuisine est importante. On apprend à calculer le coût d'un repas familial, à acheter et cuisiner des produits bio (en relation avec les paniers bio marseillais), à préparer des buffets pour plusieurs dizaines de personnes et à faire le service, à participer à la cuisine associative, etc. Cette formation peut déboucher sur des projets de créations d'entreprises de restauration, d'insertion professionnelle dans des structures existantes. Il en va de même pour la couture et la retouche. On met en valeur la confection d'origine puisqu'il y a des débouchés réels avec les différentes communautés ethniques (vêtement pour le mariage, les fêtes familiales, etc.). Il y a aussi des débouchés économiques dans les différents marchés (marché aux puces de Noailles, marché du Soleil).

On le voit, l'insertion sociale et professionnelle est au cœur de ce type d'association. L'apprentissage de la langue est important mais n'a de sens que par rapport à un projet de vie personnel ou collectif. On est dans la formation solidaire à la fois formelle mais aussi informelle. L'offre institutionnelle est bien trop normative, avec pour conséquence d'exclure ce type de pédagogie de l'accompagnement et de la solidarité. 


\subsection{Deuxième bilan : prendre en compte le migrant acteur social}

Ces exemples montrent que le circuit officiel pour les migrants ne couvre pas l'ensemble des besoins réels de la migration. L'État impose de plus en plus de contrôles, que l'on nomme pudiquement « qualité », de plus en plus de rationalisation par le biais de l'audit, avec pour conséquence d'exclure un nombre significatif d'offres solidaires qui sont pourtant au plus près des réalités quotidiennes que vivent les migrants. On traite et gère l'urgence qui se réduit aux cours de langues. On oublie que chaque migrant est un acteur social porteur d'un projet, disposant d'un capital social consistant, vivant dans un milieu aux mille facettes.

\section{Migrants et société numérique : revisiter la didactique des langues}

Peut-on ignorer que les services publics sont aujourd'hui dématérialisés ? Peuton ignorer que le code écrit n'est plus la seule modalité de communication ? N'est-il pas devenu incontournable d'envisager la littératie de manière plus large en l'englobant dans une littératie numérique multimodale ? Cette nouvelle réalité demande de revisiter les fondements de la didactique.

\subsection{La sémiotique symbolique sociale}

Nous avons vu, avec Simmel et l'école de Chicago, l'importance de l'interactionnisme symbolique. Chaque personne construit un univers de sens, un univers symbolique. Le dialogisme est inhérent à ce processus de co-construction symbolique, comme l'a montré Volochinov (1977) en parlant de compréhension responsive.

Le linguiste Halliday (1975) proposait lui aussi une approche sociale de la langue et mettait en avant le « meaning potential » (le potentiel de signification) situant le langage dans le cadre plus large d'une sémiotique sociale. Le système linguistique n'est qu'un mode, parmi d'autres, d'actualisation des significations. Kress et van Leewen (2001) ont poursuivi les réflexions de Halliday pour définir la multimodalité, qui constitue aujourd'hui une caractéristique essentielle des messages que l'on produit ou lit sur la toile. Le répertoire de ressources n'est pas seulement plurilingue, il est avant tout sémiotique. La communication n'est 
possible que si l'ensemble des personnes partagent et co-construisent les éléments de cette sémiotique sociale. La question du sens multimodal est ainsi centrale, en particulier pour la toile, et ne peut plus être réduite au seul mode graphique écrit. Nous utilisons sur internet, de manière intentionnelle, différents modes pour signifier. Lebrun, Lacelle \& Boutin (2012) proposent de prendre en compte cette nouvelle " littératie médiatique multimodale » à l'école. La formation des migrants devrait également en tenir compte.

\subsection{De la compétence numérique à la société numérique inclusive}

L'exclusion sociale et l'illettrisme, dans ce monde interconnecté, ne relèvent plus uniquement de la capacité à lire, comprendre, écrire et compter. Les prochains illettrés auront certes des difficultés avec la littératie fonctionnelle mais risquent également d'être des illettrés numériques. Les instances nationales et internationales proposent depuis le début des années 2000 différentes définitions autour de la notion de littératie numérique. Nous sommes ainsi passés d'une approche purement technologique de la compétence numérique (utiliser l'ordinateur et internet) à une approche plus critique centrée sur le traitement de l'information et des médias (Unesco 2003/08). La compétence numérique est devenue une compétence clé (Parlement européen 2006) et a été reprise dans la plupart des référentiels. La Commission européenne (2007) s’intéresse à la question de la société numérique inclusive. Il ne suffit plus uniquement de compétence informatique de base mais de la capacité à résoudre des problèmes avec le numérique dans le cadre de la vie quotidienne et professionnelle. Les futurs citoyens, dont les migrants adultes, ont besoin d'une formation adaptée. Pour cela les enseignants doivent eux aussi devenir compétents en littératie numérique afin qu'ils ne deviennent à leur tour des illettrés du numérique. Prenski (2001 : 2) parlait de « digital immigrant teachers ».

Centeno (2011), lors d'un atelier sur la compétence numérique et l'inclusion sociale pour l'horizon 2020, montre l'importance d'une approche par projet et résolution de problème, c'est-à-dire une approche qui prendrait mieux en compte les situations sociales complexes. Il s'agit de s'engager dans le tournant social (Springer 2009) et de préparer l'avenir avec une approche de la globalité et de la complexité. Cette ouverture vers une société inclusive se retrouve dans différents référentiels professionnels. C'est le cas du référentiel de l'association nationale de lutte contre l'illettrisme, en France, qui a conçu en 2009 un référentiel des compétences clés en situation professionnelle. Il est intéressant de noter que ce référentiel souligne que l'employabilité, pour les personnes fragiles, 
ne peut plus se résumer au seul savoir parler, lire, écrire et compter. Il est aujourd'hui essentiel de prendre en compte non seulement la compétence en littératie numérique mais aussi la compétence sociale et l'apprendre à apprendre.

\subsection{Revisiter la didactique des langues}

Il est donc temps de revisiter la didactique des langues, ainsi que les courants de l'apprentissage, pour tenir compte de la nouvelle donne numérique. En didactique des langues, malgré l'insistance sur la plurilittératie, la littératie fonctionnelle demeure majoritairement prise en compte par les manuels et les certifications (lire, comprendre et produire un texte simple dans une langue). On vise le socle de base du code écrit, comme c'est le cas pour l'alphabétisation et les niveaux élémentaires en français langue étrangère. Une nouvelle évolution est devenue nécessaire. La littératie numérique multimodale devrait, pour les raisons évoquées, être prise en compte. Elle ne se rajoute pas à la plurilittératie mais l'englobe dans la mesure où elle permet de prendre en compte les différents modes et modalités des littératies.

D'un point de vue pédagogique, une approche projet autour de tâches sociales complexes (Springer 2010) permet de situer les apprentissages dans la complexité et le symbolisme social. L'apprentissage pourrait se faire à partir de scénarios ouverts permettant la résolution de problèmes concrets (Huver et Springer 2011). Il s'agit de ne pas rester aux niveaux taxonomiques élémentaires de reproduction et de renverser en quelque sorte la taxonomie de Bloom pour donner toute son importance à l'action sociale (figure 1).

Cette visée phénoménologique de la formation prend la globalité humaine en compte : les aspects utilitaristes du quotidien (la polarité prosaïque) mais aussi les aspects symbolique, imaginaire et poétique (la polarité poétique). L'évaluation pourrait alors rendre compte du processus expérientiel. C'est une évaluation qui rend justice aux valeurs et aux principes humanistes de l'apprentissage. Kress parle de « principles of learning » (2010 : 183) pour ce type d'évaluation de l'expérience. Il s'agit bien de rendre compte de la capacité à signifier dans différents modes et modalités. On le comprend, plurilittératie et interculturel (ce que l'OCDE nomme aujourd'hui compétence globale) sont intégrés dans cette approche du symbolisme social. Le schéma suivant (Figure 2) montre le passage d'une évaluation qui vise la conformité aux standards de la certification à une évaluation socialement partagée qui tient compte de la complexité sociale. Il s'agit dans ce cas de permettre aux participants de cartographier les compétences mises en œuvre dans les différents projets et de 


\section{Une approche alternative pour les migrants adultes}

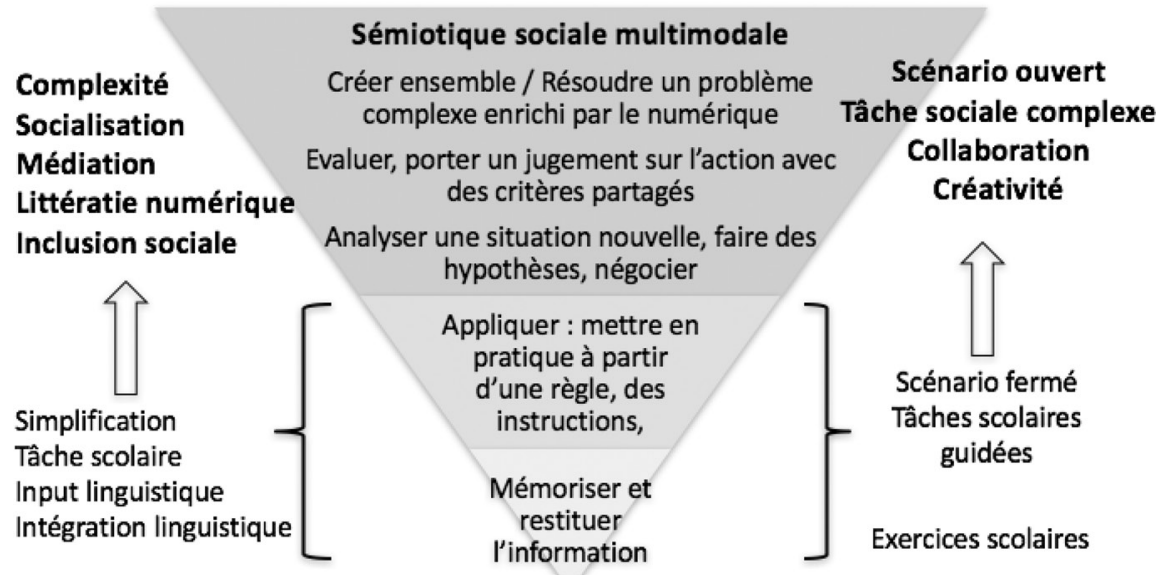

Figure 1 : Une approche alternative de la formation

référentialiser de cette manière des compétences qui dépassent les compétences linguistiques du CECR.

En ce qui concerne la validation institutionnelle, il serait tout à fait possible d'imaginer des portfolios d'apprentissage et de validation des compétences présentant les réalisations concrètes des migrants, leurs projets, tout au long d'une période, comme c'est le cas dans la procédure de validation des acquis d'expérience. Ce type d'évaluation s'intéresse à la valorisation des identités sociales en développement, à l'engagement sémiotique dans le cadre d'expériences socialement partagées, à la création d'objets culturels et de ressources multimodales.

Si nous souhaitons rendre autonomes les migrants adultes, si nous souhaitons les accompagner dans la réalisation de leur projet personnel et social, il est alors nécessaire de favoriser créativité et estime de soi (Springer 2014). Il n'est pas raisonnable d'enfermer le migrant adulte dans une pédagogie de l'urgence qui se limite aux situations et tâches de la vie quotidienne supposées définir le minimum vital pour s'intégrer dans la société d'accueil.

\section{Conclusion}

Et maintenant? Il semble que le statu quo de type formation en urgence pour le court terme s'impose et soit même renforcé partout en Europe. C'est le schéma 


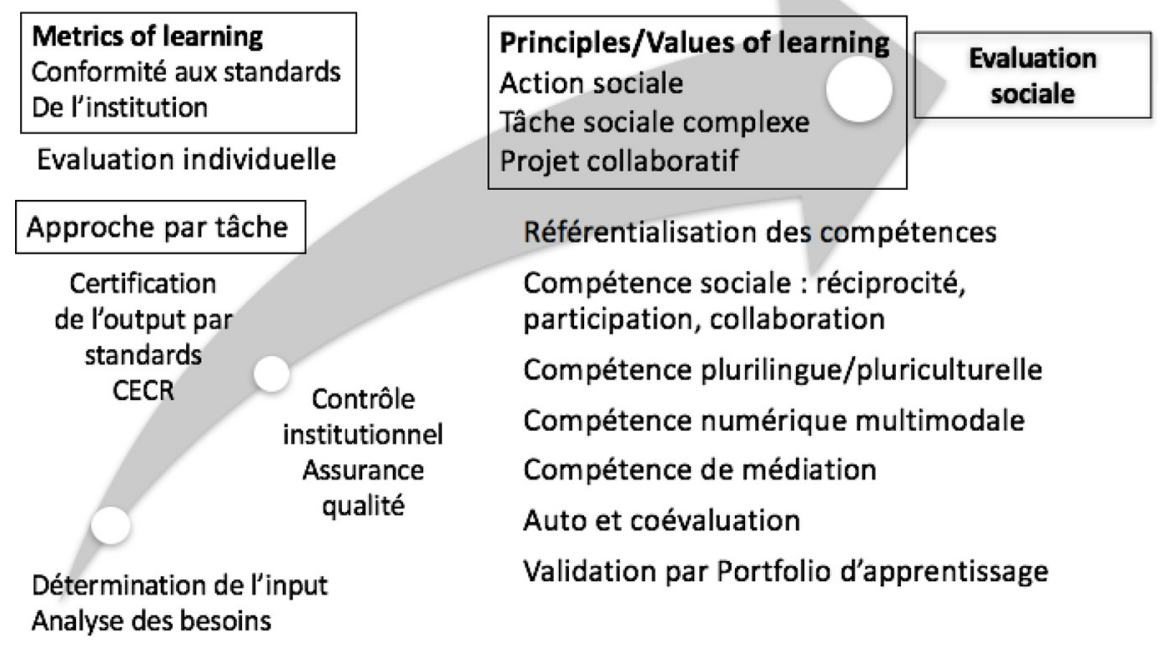

Figure 2 : D’une évaluation visant la conformité aux standards de la certification à une évaluation socialement partagée

institutionnel que nous connaissons aujourd'hui. Beaucoup de réflexions se sont focalisées sur cette offre d'urgence dans le cadre de l'ILMA. Le développement de l'e-learning, avec des plateformes institutionnelles ou commerciales, pour la formation linguistique des migrants est déjà une réalité dans plusieurs pays en Europe. Malheureusement, l'e-learning s'inscrit dans l'optique prosaïque de la survie et de l'urgence. Le risque réel est d'aboutir à une consommation de ressources linguistiques prédéfinies et à une homogénéisation de la formation avec une évaluation pour mesurer, plus facilement encore, la conformité aux standards visés (le « metrics of learning » de Kress, op. cit.).

Peut-on passer du migrant connecté à l'apprenant migrant connecté ? Il faudrait pour cela dépasser le court terme et s'engager dans le moyen et long terme. Proposer des formations d'accompagnement à moyen et long terme pourrait permettre le développement de projets pour une insertion/inclusion sociale. Le curriculum, dans ce cas, ne peut être qu'un curriculum situé et non pas prescrit. Il est défini par les acteurs eux-mêmes, au fur et à mesure des développements. Les besoins qui émergent des projets ne sont pas que langagiers et n'ont pas à être dictés technocratiquement par une ingénierie externalisée. L'évaluation sociale et collaborative pourrait alors s'inscrire dans une valorisation des expériences et des compétences multiples, selon les principes et les valeurs de l'apprentissage. La certification externe n'a pas de sens dans ce contexte. 
La formation des différents formateurs est un réel souci. Le référentiel de l'Unesco (2011) propose des pistes intéressantes pour la formation des enseignants afin d'intégrer le numérique et l'approche projet. Cependant, ces évolutions ne seront possibles que si les décideurs sont sensibilisés à ces problèmes et convaincus de l'intérêt de faire évoluer la vision de la formation des migrants ainsi que les pratiques formatives. Il est urgent de donner une plus grande marge de manœuvre aux acteurs de terrain et aux apprenants migrants pour que se réalise le souhait d'une société apprenante et inclusive qui figure pourtant dans tous les textes politiques.

\section{Références}

Arp, Jean Hans. 2014. La Grande Fête sans fin. Editions Arfuyen.

Agence nationale de lutte contre l'illettrisme. 2009. Référentiel des compétences clés en situation professionnelle (RCCSP). http://www.anlci.gouv.fr/Mediatheque/Entreprises/ Entreprise/Referentiel-des-competences-cles-en-situation-professionnelle-RCCSP (consulté le 10 février 2016).

Bashi, Vilna Francine. 2007. Survival of the knitted. Immigrant social networks in a stratified world. Stanford : Stanford University Press.

Centeno, Clara. 2011. Digital competencies and inclusion. JRC European Commission. http:// is.jrc.ec.europa.eu/pages/documents/2011_05_23_DigitalCompetenceelnclusion_v4.pdf (consulté le 12 février 2016).

Commission européenne. 2007. Initiative européenne i2010 sur l'insertion numérique. http:// eur-lex.europa.eu/legal-content/FR/TXT/HTML/?uri=CELEX:52007DC0146\&from=FR (consulté le 20 janvier 2016).

Diminescu, Dana. 2005. Le migrant connecté : pour un manifeste épistémologique. Migrations société 102. 275-292.

Granovetter, Mark. 1973. The Strength of Weak Ties. American Journal of Sociology. 78(6), 1360 -1380. https://sociology.stanford.edu/sites/default/files/publications/the strength_of_weak_ties_and_exch_w-gans.pdf (consulté le 15 janvier 2016).

Halliday, Michael Alexander. 1975. Learning how to mean: explorations in the development of language. London : Edward Arnold.

Huck, Dominique. 1989. André Weckmann, écrivain de son temps. Strasbourg : Centre régional de documentation pédagogique.

Huver, Emmanuelle \& Claude Springer. 2011. L'évaluation en langues. Paris : Didier.

Kress, Gunther. 2010. Multimodality: A Social Semiotic Approach to Contemporary Communication. New York : Routledge.

Kress, Gunther \& Theo van Leeuwen. 2001. Multimodal Discourse: The Modes and Media of Contemporary Communication. Oxford: Oxford University Press

Lebrun, Monique, Nathalie Lacelle \& Jean-François Boutin. 2012. La littératie médiatique multimodale. De nouvelles approches en lecture-écrire à l'école et hors de l'école. Québec : Presses de l'Université du Québec.

Maalouf, Amin. 1998. Les identités meurtrières. Le Livre de Poche. 
Morin, Edgar. 2015. Penser global. L'humain et son univers. Paris : Robert Laffont.

Parlement Européen. 2006. Journal officiel de l'Union européenne. 2006. http://eur-lex. europa.eu/LexUriServ/LexUriServ.do?uri=0J:L:2006:394:0010:0018:fr:PDF (consulté le 20 janvier 2016).

Peraldi, Michel, Claire Duport \& Michel Samson. 2015. Sociologie de Marseille. Paris : La Découverte.

Prensky, Marc. 2001. Digital natives, digital immigrants. On the horizon 9.5, 1-6.

Raphaël, Freddy. 2008. Le juif comme paradigme de l'étranger dans l'œuvre de G. Simmel. Sociétés 3 ( $\left.n^{\circ} 101\right)$. 81-90. https://www.cairn.info/revue-societes-2008-3-page81.htm (consulté le 25 mai 2016).

Simmel, Georg. 1979 [1908]. Digressions sur l'étranger. In Yves Grafmeyer \& Joseph Isaac (eds.), L'École de Chicago, naissance de l'écologie urbaine, 53-77. Paris : Ed. Du Champ urbain.

Siemens, George. 2004. Connectivism: A learning theory for the digital age. International Journal of Instructional Technology and Distance Learning. http://www.itdl.org/Journal/ Jan_05/article01.htm (consulté en octobre 2015).

Springer, Claude. 2009. La dimension sociale dans le CECR : pistes pour scénariser, évaluer et valoriser l'apprentissage collaboratif. In Evelyne Rosen. La perspective actionnelle et l'approche par les tâches en classe de langue. Le français dans le monde. Recherches et applications, $\mathrm{n}^{\circ} 45.25-34$.

Springer, Claude. 2010. Les projets collaboratifs (TIC) : quelles compétences pour quelle évaluation ? In Actes du Congrès International : Communiquer avec les langues cultures. Thessalonique : University Studio Press, 516-528.

Springer, Claude. 2014. La question de l'évaluation en formation des enseignants de langues : de l'évaluation de la performance à l'évaluation de la collaboration créative. Actes du colloque Créativité et apprentissage : un tandem à réinventer ? https://hal. archives-ouvertes.fr/hal-01300903/document (consulté le 20 février 2016).

Unesco. 2003. Déclaration de Prague : Vers une société compétente dans l'usage de l'information. http://www.enssib.fr/bibliotheque-numerique/documents/1900declaration-de-prague-vers-une-societe-competente-dans-l-usage-de-l-information.pdf (consulté le 20 janvier 2016).

Unesco. 2008. Programme Information pour tous (PIPT). Vers des indicateurs de la maitrise de l'information. http://www.uis.unesco.org/Library/Documents/wp08_infolit-fre.pdf (consulté le 20 janvier 2016).

Unesco. 2011. Un référentiel de compétence pour les enseignants. http://unesdoc.unesco.org/ images/0021/002169/216910f.pdf (consulté en avril 2014).

Vigée, Claude. 2000. Les Orties Noires. Schwarzi Sengessle. Un requiem alsacien. Oberlin. Volochinov, Valentin Nikolevitch. 1977. Le marxisme et la philosophie du langage. Paris : Éditions de Minuit. 